\title{
Special Issue: Membrane and Receptor Dynamics
}

\author{
Shikha Prakash ${ }^{1} \cdot$ Durba Sengupta $^{1}$
}

Published online: 3 October 2019

(c) Springer Science+Business Media, LLC, part of Springer Nature 2019

The cell membrane is the final frontier of the cell and determines its identity and communication strategies with the outside world (Edidin 2003). The diversity of the lipids, sterols, and sugar moieties underlines the critical role of the membrane in both, large-scale behavior such as providing structure and barriers and in the fine tuning nano-scale behavior of membrane protein function. The dynamics of the membrane and the associated proteins is a complex interplay spanning multiple spatial and temporal hierarchies (see Fig. 1). The current issue includes a collection of twentyone articles, from experts in the field, that explore the key features of cell-membrane dynamics by focusing on human health and disease. The issue can be broadly divided into (i) membrane dynamics, (ii) membrane-peptide interactions, (iii) protein dynamics and organization, with a special focus on GPCRs. These aspects have been probed by both experimental and computational approaches, and provide complementary insights at different spatio-temporal resolutions. Taken together, these articles highlight the complexity of membrane organization and dynamics and its relation to myriad cellular outcomes. We hope this issue will initiate more cross-talk within the communities and help unravel further mysteries of this complex cross-talk.

Seminal works on model membranes have provided insights into the physico-chemical principles underlying membrane dynamics (Nicolson 2014), but its relation to live cells has been famously complicated, in part due to the limited resolution of current techniques. The review by Adhyapak and Kapoor (2019) provides an overview on the lateral heterogeneity and dynamic cross-talk in cell membranes and recaps how physico-chemical properties control the cellular function. They compare specific and non-specific effects to show how these lead to a fine modulation of protein-lipid interactions, and highlight their relevance in diseased

\section{Durba Sengupta \\ d.sengupta@ncl.res.in}

1 CSIR-National Chemical Laboratory, Dr. Homi Bhabha Road, Pune 411 008, India conditions such as cancer and infectious diseases. They argue that altered lipid dynamics could serve as a potential biomarker for cancers and perhaps modulating lipid dynamics could be the new frontier in lipid-based therapeutics. As indicated above, one of the challenges in probing lipid dynamics is the lack of consistencies in the spatio-temporal scales of the experimental and computational approaches. For instance, fluorescent probes are used to label lipids to investigate the structural and dynamic properties of membrane via optical microscopy techniques. Though the effects of fluorescent probes are assumed to be too small to consider (Maier et al. 2002; Haldar and Chattopadhyay 2012), the optically labeled lipid could change the local lipid structure and lateral mobility. Brancanto and co-workers (2019) have used atomistic molecular dynamics simulations to compare temperature-dependent structural and dynamic changes in model DOPC membranes with standard Rhodamine B-labeled DOPE lipids. They report small but distinct differences arising in at different temperatures such as in diffusion and permeation. These results indicate that the differential membrane anisotropy could have implications for optical microscopy studies of lipid membranes. Another aspect of membrane dynamics that is often ambiguously defined and difficult to probe is membrane permeability. Cunill-Semanat and Salgado (2019) provide an overview of how theoretical, computational, and experimental studies can provide insights into the mechanisms of pore opening and closing and the main intermediates involved. They argue that lipid mechanics is an integral factor, and redefinition of pore edge energy from computational methods gives new insight into distinctive breakdown regimes known from experiments.

Membrane fusion and, its seemingly counter phenomenon, fission are areas where membrane dynamics is intricately coupled to protein dynamics, especially to overcome the energy barriers along complicated pathways. The role of fusion peptides that drive the structural changes and in turn the role of lipid composition in modulating fusion peptides is crucial for understanding the complete pathway. In the article by Meher and Chakraborty (2019), proteins that are involved in membrane fusion are reviewed. They highlight 


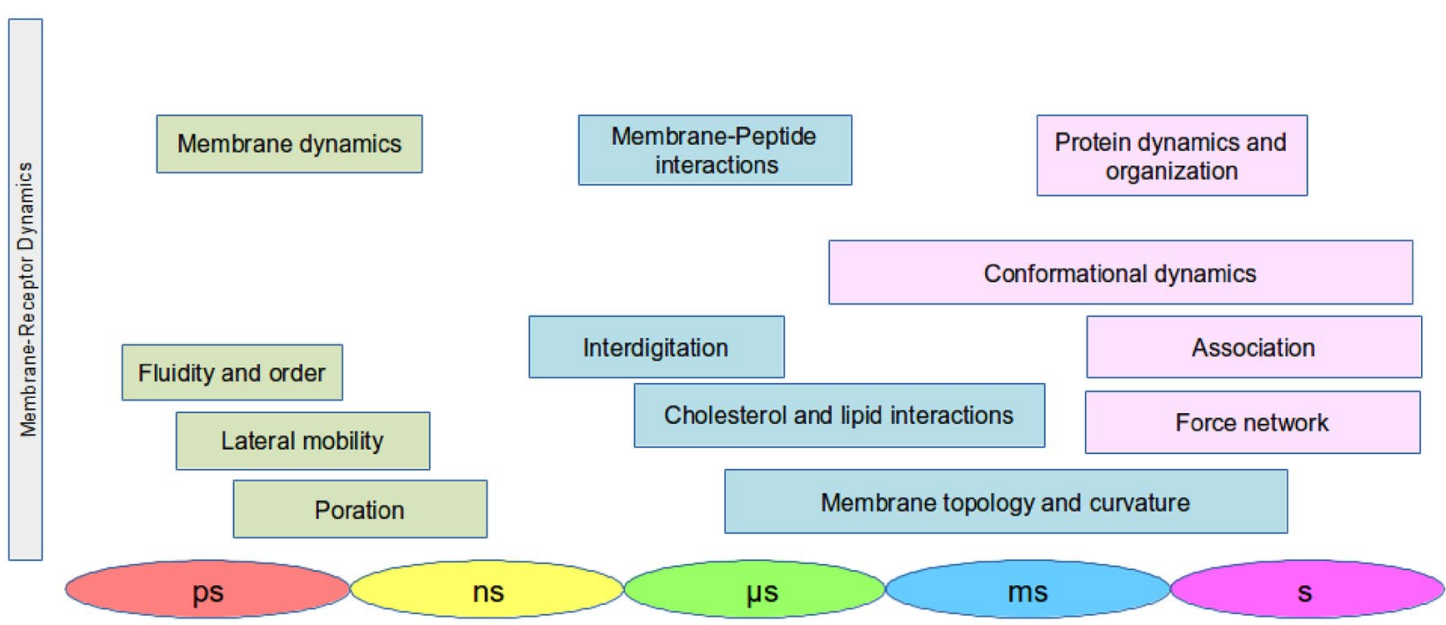

Time Scale

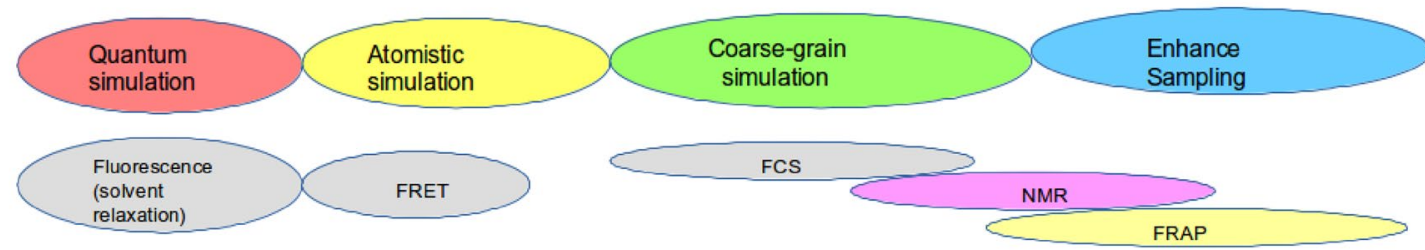

Fig. 1 An overview of the time-scales associated with various aspects of membrane and receptor dynamics, focusing on the methodologies and approaches reported in this special issue

three categories, docking proteins that select which membranes to fuse, regulatory proteins that control the process, and fusion proteins that finally fuse the lipid bilayers. The review investigates the current status of direct and indirect lipid effects and interactions of fusion peptides. Interestingly, in several cases intrinsically disordered proteins and protein regions (IDPRs) do not interact directly with membranes, but they play a major role in regulating the conformational changes in proteins involved in membrane remodeling. The paper by Appadurai et al. (2019) highlights the sequence-disorder-function with a focus on structural landscape of IDPRs and how conformational flexibility is related to membrane curvature dynamics. Fusion in organelles is an important aspect in the context of membrane remodeling and lipid composition effects. Taly and co-workers have recently used molecular modeling approaches to analyze the determinants of membrane curvature and the first steps of mitochondrial fusion. They report here a general model using data from experimental and computational methods to explain our current understanding of membrane fusion events (De Vecchis et al. 2019). Membrane fission is an emerging area where mechanical properties of the membrane are intricately balanced to signaling pathways and endocytotic machineries. In the paper by Scarlata and co-workers (2019), they investigate the impact of stretch on the membrane, especially the dynamics of stretch on $\mathrm{G}$ protein-coupled receptor
(GPCR)-G protein $(\mathrm{Gq})$ protein signaling. They observe that muscle cell stretching reduces the curvature of caveolar membrane domains by increasing the GPCR-Gq signal and modulates the calcium levels that play a key role in muscle toning and contraction.

Several peptides interact with the membrane by only one of the membrane leaflets but are able to modulate and dictate membrane dynamics in complex ways. Here, Shahane et al. (2019) report the interaction between synthetic cationic antimicrobial lipopeptides (AMLPs) and model bacterial lipid bilayers using the microsecond-timescale atomistic molecular dynamics simulations. Synthetic AMLPs are gaining attraction because unlike traditional antibiotics which target specific proteins and have a high risk of inducing bacterial resistance, the synthetic AMLPs indirectly interact with proteins and perturb the cell membrane directly. Orsi and co-workers report the effect of AMLP concentration on structural, mechanical, and dynamical features of the membrane. In particular, they observe that at higher concentrations, these lipopeptides are able to cause membrane disruption and interdigitation. Membrane interdigitation appears to be a mode of action of several peptides. The study by Vemparala et al. (2019) reports that at temperatures close to the transition temperature of membrane, Neurite outgrowth inhibitor protein-66 (Nogo-66) initiates interdigitation of lipids in opposing leaflets. Their results suggest that 
temperature plays a regulatory role in interactions between membrane-active proteins and the membrane including inducing interdigitated phases. Membrane peptides may also exhibit specific lipid-dependent behavior as exemplified in the study by Christensen and Schiott (2019). The decay of insulin-producing beta cells is correlated with the formation of amyloids by human islet amyloid polypeptides (hIAPP) in type II diabetes mellitus. Interestingly, they observe the formation of nanodomains in a mixed bilayer in the presence of hIAPP. They propose a dual role of the gangliosides on hIAPP aggregation and a direct influence of binding mode on secondary structure of peptides.

A recurring theme that is being increasingly reported is organization of receptors within membranes and the role of membrane lipids in organization (Sarkar and Chattopadhyay 2019; Sengupta et al. 2018). This special issue includes several examples focusing on different cell surface receptors. In the article by Dixon and co-workers (2019), an important role of cholesterol concentration on the association of Neu receptors is reported. Neu receptors are a sub-family of receptor tyrosine kinases that regulate cell growth, proliferation, and differentiation and are linked to a number of human cancers, including an aggressive form of breast cancer. Dixon and co-workers have used high-resolution magic angle spinning solid-state NMR and report on a unique modulation of transmembrane helix-helix interactions. The study assumes added significance in light of altered lipid composition in several cancers (Pawar and Sengupta 2018) and the large conformational heterogeneity reported previously (Pawar and Sengupta 2019). Similarly, Bechinger and co-workers (2019) investigate the structure and topology of the heterodimer the major histocompatibility complex (MHC II), implicated in autoimmune diseases like diabetes type I using a combination of CD and solid-state NMR spectroscopy. They show that MHC II heterodimers consisting of DQ $\alpha 1$ (DQA1) and DQ $\beta 1$ (DQB1) interact via GxxxG motifs and are mediated interactions. They report for the first time the effect of sphingomyelin on the structure of these heterodimers. Even for large receptor complexes such as the nicotinic acetylcholine receptor (nAChR), the structure and function has been reported to be regulated by lipid effects, although the molecular mechanisms underlying cluster formation are currently not very clear. Woods et al. (2019) have investigated the nAChR interactions in domain-forming acidic lipid membranes via coarse-grained molecular dynamics simulations. The results suggest that protein concentration plays a deciding role in $\mathrm{nAChR}$ oligomerization in lipid domains. Decision making by immune receptors to select specific signals over others is an active field due to its significance in immune therapy and biotechnological applications. The current review by Limozin and Puech (2019) investigates the role of altering forces in the organization and function of immune receptors. They have compared receptors present at the surface of lymphoid cells, such as B and T lymphocytes and Fc receptors of natural killer (NK) cells and describe how the cell membrane and membrane-cytoskeleton interactions exert a force on these receptors and in turn regulate their function. The review highlights the importance of considering and screening large fluctuations in scale that are at play in the process leading from protein interaction and membrane apposition. Their recent work Wahl et al. (2019) and from several other groups suggests that the role of the cytoskeletal effects on receptor organization needs further investigation (Prakash et al. 2019; Deshpande et al. 2017). The supramolecular organization of rhodopsin within the membrane of photoreceptor cells is reviewed in the article by Park (2019). The aggregation of rhodopsin and lipid effects assume significance since it can lead to retinal degeneration. In this context, the article by Brown and co-workers (2019) provides an exhaustive report on the sub-atomic, atomistic, and molecular scale dynamics of rhodopsin function.

At the far end of the complexity scale, membrane-embedded multi-span proteins are modulated and sometimes dictated by membrane dynamics. An example of specific protein-lipid interactions is reported by Albano et al. (2019), where they investigate the effect of calcium ions on the binding and transport properties of a Cx26 connexon proteins. The connexin family of structural proteins constitutes the inter-cellular membrane channels, called gap junctions. The authors identify specific amino acids that form hydrogen bonds with the POPC lipids and play a critical role in protein-lipid interactions. Similar protein-lipid interplay could be crucial in the case of transporter proteins such as acid resistance antiporters. Krammer and Prevost (2019) provide a comprehensive overview of the structural and dynamic features of the superfamily. A detailed correlation between the structural and mechanistic features of these antiporters will help delineate the origin of substrate selectivity. With the focus on structural domains, the extra-membrane regions that are often intrinsically disordered as well as post-translationally modified have not until recently been explored much. The article by Pal and Chattopadhyay (2019) highlights the need to investigate the disordered extramembranous regions in GPCRs, which would be important to develop a comprehensive understanding of GPCR structure, function, and dynamics. The extracellular and intracellular loops (ECLs and ICLs) have been discussed in depth giving a unique perspective. They concluded that further understanding of ECLs and ICLs would give better insights to develop improved therapeutics. This is exemplified in the paper by Bhosale et al. (2019), where they focus on $\beta_{2^{-}}$ adrenergic receptor $\left(\beta_{2} \mathrm{AR}\right)$. They have performed atomistic simulations to explore the molecular mechanism underlying the altered drug response in the 27 th position population variation. They conclude that the glutamic acid residue induces 
electrostatic perturbations that propagate to the binding site. The findings could lead to the improved design of genotypespecific agonists for the $\beta_{2} \mathrm{AR}$ and mark a first step towards precision medicine for asthma and COPD targeting the multiple population variants reported (Tandale et al. 2016). The final article in the special issue is a comprehensive overview by Dakshinamurti and co-workers (2019) on post-translational modifications in GPCRs. Of special interest to the readers will be the effect of palmitoylation in extracellular disordered domains that would directly result in cross-talk between GPCRs and membrane domains.

In conclusion, we have now identified a few organizational principles underlying membrane and receptor dynamics. The new frontiers in this regard are integrating the spatio-temporal scales, providing a molecular handle to large flexible domains, and cytoskeletal effects exerted on receptor and oligomer complexes. One could imagine considering proteins and lipids to be one supramolecular entity with a coupled dynamics. These are exciting times ahead for membrane biology with inputs from a range of complementary fields including structural biology, computational biology, and spectroscopy.

Acknowledgements The authors thank their group members for discussions. SP gratefully acknowledges the Dept. Biotechnology (DBT, Govt. of India) for the BioCARe grant and DS acknowledges the Dept. Science Technology (DST, (Govt. of India) Grant: EMR/2016/002294) for funding. We thank the authors of the special issue and Prof. A. Ladokhin and Prof. A.N. Bondar for the opportunity and help.

\section{References}

Adhyapak P, Kapoor S (2019) Membrane dynamics in health and disease: the journey so far. J Membr Biol. https://doi.org/10.1007/ s00232-019-00087-0

Aisenbrey ES, Salnikov C, Bechinger B (2019) Solid-state nmr investigations of the mhc ii transmembrane domains: topological equilibria and lipid interactions. J Membr Biol. https://doi.org/10.1007/ s00232-019-00071-8

Akhunzada MJ, Sagresti L, Catte A, Bhattacharjee N, Agostino TD, Brancato G (2019) Temperature dependence of the structure and dynamics of a dye-labeled lipid in a planar phospholipid bilayer: a computational study. J Membr Biol. https://doi.org/10.1007/s0023 2-019-00081-6

Albano Juan MR, Jara Gabriel E, Laura Fernndez M, Facelli Julio C, Ferraro Marta B, Pickholz Monica (2019) The effects of calcium on lipid-protein interactions and ion flux in the cx26 connexon embedded into a POPC bilayer. J Membr Biol. https://doi. org/10.1007/s00232-019-00088-z

Appadurai VN, Uversky R, Srivastava A (2019) The structural and functional diversity of intrinsically disordered regions in transmembrane proteins. J Membr Biol. https://doi.org/10.1007/s0023 2-019-00069-2

Bhosale SV Nikte, Sengupta D, Joshi M (2019) Differential dynamics underlying the gln $27 \mathrm{glu}$ population variant of the beta2adrenergic receptor. J Membr Biol. https://doi.org/10.1007/s00232-01900093-2
Christensen M, Schiott B (2019) Revealing a dual role of ganglioside lipids in the aggregation of membrane-associated islet amyloid polypeptide. J Membr Biol. https://doi.org/10.1007/s0023 2-019-00074-5

Cunill Semanat E, Salgado J (2019) Spontaneous and stress-induced pore formation in membranes: theory, experiments and simulations. J Membr Biol. https://doi.org/10.1007/s00232-019-00083 $-4$

De Vecchis D, Brandner A, Baaden M, Cohen MM, Taly A (2019) A molecular perspective on mitochondrial membrane fusion: from the key players to oligomerization and tethering of mitofusin. $\mathrm{J}$ Membr Biol. https://doi.org/10.1007/s00232-019-00089-y

Deshpande S, Pawar A, Dighe A, Athale C, Sengupta D (2017) Role of spatial inhomogeneity in GPCR dimerisation predicted by receptor association-diffusion models. Phys Biol 14:036002

Edidin M (2003) Lipids on the frontier: a century of cell-membrane bilayers. Nat Rev Mol Cell Biol 4:414

Ellis D, Brown N, Lewandowski SP, Hasan JR, Patel M, Dixon AM (2019) Modulation of transmembrane domain interactions in neu receptor tyrosine kinase by membrane fluidity and cholesterol. J Membr Biol. https://doi.org/10.1007/S00232-019-00075-4

Garwain O, Qifti A, Scarlata S (2019) Mechanical stretch redenes membrane GPCR-Gq-calcium signaling complexes. J Membr Biol. https://doi.org/10.1007/s00232-019-00063-8

Haldar S, Chattopadhyay A (2012) Application of NBD-labeled lipids in membrane and cell biology. In: Mély Y, Duportail G (eds) Fluorescent methods to study biological membranes. Springer series on fluorescence (methods and applications), vol 13. Springer, Berlin

Krammer E-M, Provos M (2019) Function and regulation of acid resistance antiporters. J Membr Biol. https://doi.org/10.1007/s0023 2-019-00073-6

Limozin L, Puech P-H (2019) Membrane organization and physical regulation of lymphocyte antigen receptors: a biophysicists perspective. J Membr Biol. https://doi.org/10.1007/s00232-01900085-2

Maier O, Oberle V, Hoekstra D (2002) Fluorescent lipid probes: some properties and applications (a review). Chem Phys Lip 116:3-18

Meher G, Chakraborty H (2019) Membrane composition modulates fusion by altering membrane properties and fusion peptide structure. J Membr Biol. https://doi.org/10.1007/s00232-019-00064-7

Nicolson GL (2014) The fluid mosaic model of membrane structure: Still relevant to understanding the structure, function and dynamics of biological membranes after more than 40 years. Biochim Biophys Acta 1838:1451-1466

Pal S, Chattopadhyay A (2019) Extramembranous regions in G proteincoupled receptors: Cinderella in receptor biology? J Membr Biol. https://doi.org/10.1007/s00232-019-00092-3

Park P-S-H (2019) Rhodopsin oligomerization and aggregation. J Membr Biol. https://doi.org/10.1007/s00232-019-00078-1

Pawar AB, Sengupta D (2018) Effect of membrane composition on receptor association: implications of cancer lipidomics on erbb receptors. J Membr Biol 251:359-368

Pawar AB, Sengupta D (2019) Resolving the conformational dynamics of erbb growth factor receptor dimers. J Struct Biol 207:225-233

Prakash S, Krishna A, Sengupta D (2019) Actin cytoskeleton and membrane interactions: role in GPCR function and organization. Proc Indian Natl Sci Acad 85:1-12

Ryazantsev MN, Nikolaev DM, Struts AV, Brown MF (2019) Quantum mechanical and molecular mechanics modeling of membraneembedded rhodopsins. J Membr Biol. https://doi.org/10.1007/ s00232-019-00095-0

Sarkar P, Chattopadhyay A (2019) Exploring membrane organization at varying spatiotemporal resolutions utilizing fluorescence-based approaches: implications in membrane biology. Phys Chem Chem Phys 21:11554-11563 
Sengupta D, Prasanna X, Mohole M, Chattopadhyay A (2018) Exploring GPCR-lipid interactions by molecular dynamics simulations: excitements, challenges, and the way forward. J Phys Chem B 122:5727-5737

Shahane G, Ding W, Palaiokostas M, Azevedo HS, Orsi M (2019) Interaction of antimicrobial lipopeptides with bacterial lipid bilayers. J Membr Biol. https://doi.org/10.1007/s00232-019-00068-3

Sikarwar AS, Bhagirath AY, Dakshinamurti S (2019) Effects of posttranslational modications on membrane localization and signaling of prostanoid gpcrg protein complexes and the role of hypoxia. J Membr Biol. https://doi.org/10.1007/s00232-019-00091-4

Tandale A, Joshi M, Sengupta D (2016) Structural insights and functional implications of inter-individual variability in $\beta 2$-adrenergic receptor. Sci Rep 6:24379

Vemparala S, Devanand S, Krishnaswamy T (2019) Interdigitation of lipids induced by membraneactive proteins. J Membr Biol. https ://doi.org/10.1007/s00232-019-00072-7
Wahl A, Dinet C, Dillard P, Nassereddine A, Puech P-H, Limozin L, Sengupta K (2019) Biphasic mechanosensitivity of t cell receptor-mediated spreading of lymphocytes. Proc Natl Acad Sci USA 116:5908-5913

Woods L, Sharp K, Brannigan G (2019) Untangling direct and domain mediated interactions between nicotinic acetylcholine receptors in DHA-rich membranes. J Membr Biol. https://doi.org/10.1007/ s00232-019-00079-0

Publisher's Note Springer Nature remains neutral with regard to jurisdictional claims in published maps and institutional affiliations. 